\title{
O TRABALHO GERENCIAL DO ENFERMEIRO NA REDE BÁSICA DE SAÚDE
}

\author{
Claci Fátima Weirich ${ }^{1}$, Denize Bouttelet Munari², Silvana Martins Mishima³, Ana Lúcia Queiroz Bezerra ${ }^{4}$
}

\footnotetext{
${ }^{1}$ Doutora em Ciências da Saúde. Professora da Faculdade de Enfermagem da Universidade Federal de Goiás (UFG). Goiás, Brasil. E-mail: claci@cultura.com.br

${ }^{2}$ Doutora em Enfermagem. Professor Titular da Faculdade de Enfermagem da UFG. Goiás, Brasil. E-mail: denize@fen.ufg.br

${ }^{3}$ Doutora em Enfermagem. Professor Titular do Departamento Materno Infantil e Saúde Pública da Escola de Enfermagem de Ribeirão Preto da Universidade de São Paulo. São Paulo, Brasil. E-mail: smishima@eerp.usp.br

${ }^{4}$ Doutora em Enfermagem. Professora da Faculdade de Enfermagem da UFG. Goiás, Brasil. E-mail: aqueiroz@fen.ufg.br
}

\begin{abstract}
RESUMO: Este estudo focaliza questões da gerência nos serviços públicos de saúde e têm como objetivo identificar elementos do trabalho gerencial do enfermeiro na Rede Básica de Saúde de Goiânia-GO. Pesquisa exploratória descritiva, de base quantitativa, realizada entre 2006/2007. Para a coleta de dados foi utilizada a entrevista estruturada com 44 enfermeiros gerentes de serviços. Foi construído um banco de dados, utilizando o Software Epi-Info versão 3.3.2. Os dados foram submetidos a um processo de codificação e analisados por medida de frequência simples e nas variáveis que o enfermeiro pode discorrer utilizamos a categorização por semelhança. Os resultados apontam aspectos que fortalecem e tornam mais visíveis as ações gerenciais dos enfermeiros, ao mesmo tempo que mostram as fragilidades do trabalho gerencial na Rede Básica de Saúde. A contribuição dos enfermeiros gerentes foi de grande significado para que a formação seja repensada pelas universidades e pelos gestores.
\end{abstract}

DESCRITORES: Gestão em saúde. Enfermagem em saúde pública. Atenção primária à saúde. Enfermagem.

\section{MANAGERIAL WORK OF BRAZILIAN FEDERAL HEALTH CARE SYSTEM NURSES}

\begin{abstract}
This study focuses on management issues in public health services and attempts to identify elements of nursing managerial work in the local Public Health Care system of Goiânia-GO, Brazil. This exploratory, descriptive and quantitative study was carried out between 2006 and 2007. A structured interview was used for data collection and was composed of 44 service-manager nurses. A database was built, using the Epi-Info Software 3.3.2. The data was submitted to a coding process and analyzed through measuring simple frequencies similarity categorization. The results point out aspects which strengthen nursing managerial actions and make them more visible, as well as show the fragilities of managerial work in Brazilian Public Health Care. The contribution of these nurse managers meant a great deal in order that formal nursing education may be rethought by universities and managers.
\end{abstract}

DESCRIPTORS: Health management. Public health nursing. Primary health care. Nursing.

\section{LA GESTIÓN DEL ENFERMERO EN LA RED DE SALUD BÁSICA}

RESUMEN: Este estudio se centra en cuestiones de gestión en los servicios salud pública, teniendo como objetivo identificar elementos de la gestión del enfermero en la Red de Salud Básica del municipio de Goiânia-GO. Investigación exploratoria, descriptiva de base cuantitativa, realizada entre 2006 y 2007. Para la recolección de los datos se utilizó la entrevista estructurada con 44 enfermeros gerentes de servicios. Fue construido un banco de datos, utilizando el Software Epi-Info versión 3.3.2. Los datos fueron sometidos a un proceso de codificación y analizados por medida de frecuencia simple y la clasificación por similitud. Los resultados señalan aspectos que fortalecen y hacen más visibles las acciones de gestión de los enfermeros, al mismo tiempo que muestran las fragilidades del trabajo de gestión en la Red Básica de Salud. La contribución de los enfermeros gerentes fue de gran significado para que la formación sea repensada por las universidades y por sus gestores.

DESCRIPTORES: Gestión en salud. Enfermería en salud pública. Atención primaria de salud. Enfermería. 


\section{INTRODUÇÃO}

A expansão de Rede de Atenção à Saúde, impulsionada pelo processo de descentralização no Sistema Único de Saúde (SUS), vem operar mudanças na gestão e prestação de serviços no setor, transformando o mercado de trabalho em saúde. Neste sentido, a Enfermagem tem participado significativamente desse processo, pela ampliação da Estratégia Saúde da Família. ${ }^{1}$

Pesquisadores desta temática apontam para a necessidade de discussão e análise da gerência dos serviços locais de saúde, pois estas são as unidades produtoras, a porta de entrada no sistema local de saúde, onde os problemas podem ser identificados, priorizados e atendidos pela equipe de saúde, sendo que a gerência pode definir mudanças no modo de fazer saúde. ${ }^{2}$

Nos serviços de saúde, a qualidade deve ser enfatizada, principalmente porque o cuidado prestado ao usuário é consumido durante a sua produção, tornando-o diferente da produção de bens, em que é possível separar o produto com defeito sem maiores conseqüências. Ocorre igualmente no trabalho de enfermagem, como nos demais serviços de saúde, à medida que os bens são produzidos são consumidos no ato da produção, não podendo ser estocados e comercializados posteriormente. ${ }^{3}$

Em tal contexto, espera-se que o enfermeiro seja capaz de desempenhar um papel de gerente nos serviços de saúde, dentro de uma perspectiva participativa, onde o objetivo é alcançado pelo esforço coletivo e não pela união de esforços individuais. ${ }^{45}$

Contudo, a definição da função gerencial permanece ainda um tanto ambígua, afirmando que ela contrasta com outras funções produtivas passíveis de definições claras sobre atribuições funcionais e que condicionam comportamentos normalmente previsíveis e rotineiros. ${ }^{6}$

Dentre as experiências consideradas bem sucedidas, têm-se os municípios mineiros de Betim e de Luz, cujo modelo de gestão propôs uma estrutura organizacional horizontalizada. Seus processos decisórios funcionam a partir de colegiados formados pelas unidades de trabalho e a efetiva participação popular em Conferências Municipais de Saúde, propondo mudanças no modelo assistencial para a saúde. O reconhecimento popular veio não só em votos na eleição municipal, mas na efetiva participação nas instâncias de controle social. ${ }^{7-8}$

Considerando o panorama atual do trabalho em saúde, reconhecemos que o desenvolvimento da competência do enfermeiro para a ação geren- cial é uma tarefa complexa, principalmente pela tendência da formação, que na maioria das escolas ainda tem o foco voltado para a excelência do desenvolvimento técnico. ${ }^{9-10}$

A função gerencial no trabalho do enfermeiro é definida nas Diretrizes Curriculares Nacionais como uma ferramenta indispensável que o auxilia no seu cotidiano e nas expectativas do mercado de trabalho, principalmente dentro da perspectiva de consolidação do SUS. ${ }^{11}$

Vale lembrar, ainda, que para o desenvolvimento do processo gerencial nos serviços de saúde, o enfermeiro deve estar em sintonia com as atuais diretrizes operacionais organizadas via Pacto pela Saúde, que foram definidas em três dimensões: Pacto em Defesa do SUS, Pacto pela Vida e Pacto de Gestão. E entre as prioridades discutidas estão definidas o compromisso com o SUS e seus princípios; o fortalecimento da Atenção Básica; a valorização da saúde e a necessária articulação intersetorial; o fortalecimento do papel dos Estados; a luta pela regulação da Emenda 29 e por mais recursos financeiros para a área da saúde. ${ }^{12}$

Dentre as habilidades gerenciais que devem ser construídas na experiência cotidiana está o gerenciamento de recursos humanos que se caracteriza como uma atividade importante para a viabilização das práticas para administrar o trabalho das pessoas, ${ }^{13}$ assim como a competência interpessoal que auxilia o enfermeiro gerente no enfrentamento de desafios em seu cotidiano, principalmente a falta de confiança que o ser humano tem em seu semelhante, a insegurança e, muitas vezes, a falta de empatia, o que torna as relações superficiais e pouco produtivas. ${ }^{14}$

A gerência, portanto, deve ser entendida como atribuição dos dirigentes, trabalhadores e usuários na perspectiva de construção de um projeto que atenda às necessidades da população e que esteja voltado para a integralidade num processo cotidiano como proposta de mudança.

Este estudo focaliza questões relacionadas à gerência nos serviços públicos de saúde e têm como objetivo identificar elementos do trabalho gerencial do enfermeiro na Rede Básica de Saúde (RBS) como uma forma de pensar alternativas que possibilitem a reorganização da prática gerencial e a adequação do ensino de enfermagem.

\section{METODOLOGIA}

Trata-se de uma investigação exploratória, descritiva, de base quantitativa. O método exploratório permite uma visão geral e proporciona maior 
familiaridade com o fato ou o tema, com intenção de torná-lo mais explícito. O método descritivo permite realizar descrição de um fenômeno pela maneira como os dados são coletados. ${ }^{15}$.

O estudo foi desenvolvido nos serviços da RBS da Secretária Municipal de Saúde (SMS) no município de Goiânia, capital do Estado de Goiás, sendo que a coleta foi realizada entre os anos de 2006 e 2007.

A Rede Municipal de Saúde dispõe de 92 Unidades de Saúde Pública e 119 equipes deSaúde da Família, para atendimento das necessidades de saúde da população, que estão distribuídas nos nove Distritos Sanitários (DSs) em diferentes níveis de complexidade onde enfermeiros atuam com funções gerenciais nos serviços de saúde. ${ }^{16}$

Nesse contexto de saúde, os alunos da Faculdade de Enfermagem da Universidade Federal de Goiás (UFG) desenvolvem atividades práticas e estágios curriculares com ações assistenciais, de prevenção de doenças e promoção à saúde.

A amostra do estudo foi composta pelos enfermeiros que desenvolvem funções de gerentes, diretores ou coordenadores de serviços na RBS no município de Goiânia, independentemente do tempo de exercício na função gerencial, do tempo de graduação ou de formação em nível de pósgraduação. Como critério de inclusão neste estudo, os enfermeiros deviam estar lotados em Unidades Básicas de Saúde (UBSs), Centros de Saúde (CSs), DSs ou na Secretaria de Saúde (SS) em nível central e que concordassem em participar da investigação.

A coleta de dados foi realizada entre os meses de outubro de 2006 a março de 2007 por meio de entrevista estruturada. O instrumento de coleta de dados constou de questões relacionadas à caracterização dos serviços e do trabalho gerencial do enfermeiro. As entrevistas foram agendadas por telefone com os enfermeiros, conforme disponibilidade e foram realizadas na própria unidade de saúde em períodos formais de trabalho.
Para o desenvolvimento da investigação foram observados os aspectos éticos conforme Resolução No 196/96 do Conselho Nacional de Pesquisa e aprovado pelo Comitê de Ética em Pesquisa Médica Humana e Animal do Hospital das Clínicas da UFG, sob Protocolo CEPMHA/HC/UFG No 087/2006.

Para a análise dos dados, inicialmente foi construído um banco de dados, utilizando o Software Epi-Info versão 3.3.2. e, na seqüência, foram submetidos a um processo de codificação para proceder à análise descritiva desenvolvida com base no referencial teórico. Para a análise das variáveis contínuas - idade, tempo de graduação, tempo de serviço, tempo na atual lotação e outras, foi feita uma categorização por agrupamento e, para a realização de cálculos, usamos a medida de freqüência simples. Para a análise das variáveis que, mesmo sendo estruturadas, permitiam ao enfermeiro uma resposta a respeito da qual ele pudesse discorrer, utilizamos uma categorização fundamentada na leitura das respostas e do seu agrupamento por semelhança.

Os resultados são apresentados tentando explorar ao máximo o que as respostas dos enfermeiros gerentes puderam proporcionar com o intuito de responder aos objetivos do estudo.

\section{RESULTADOS E DISCUSSÃO}

Neste estudo, trabalhamos com a totalidade dos 44 enfermeiros com função gerencial na RBS em Goiânia. Esses profissionais representam uma categoria que tem significativamente ocupado cargos gerenciais e, que foram escolhidos em função de sua representatividade no contexto onde atuam, ainda que a informação obtida nem sempre seja generalizável no que diz respeito ao conjunto dos gerentes da RBS.

O conjunto das atividades que compõem as ações desenvolvidas nas unidades de saúde gerenciadas pelos enfermeiros pode ser observado na Tabela 1.

Tabela 1 - Atividades desenvolvidas pelos enfermeiros gerentes na Rede Básica de Saúde. Goiânia - GO, 2008

\begin{tabular}{|c|c|c|}
\hline Atividades desenvolvidas & $\mathbf{f}$ & $\%$ \\
\hline Assistência à saúde nos programas do Ministério da Saúde & 28 & 63,6 \\
\hline Planejamento, supervisão e avaliação dos serviços Assessoria técnica & 20 & 45,4 \\
\hline Atividades administrativas, contratação e capacitação de Recursos Humanos e sistema de informação & 16 & 36,3 \\
\hline Educação para a comunidade / educação em saúde & 13 & 29,5 \\
\hline Educação em serviço / capacitação da equipe & 12 & 27,3 \\
\hline Atenção às urgências e emergências & 10 & 22,7 \\
\hline Assistência especializada ambulatorial & 10 & 22,7 \\
\hline
\end{tabular}


Existem algumas atividades que são comuns e executadas por quase todos os gerentes, independente de estarem lotados nas UBSs, nos CSs, nos DSs ou na coordenação de serviços da SMS.

Entre elas, destacam-se: assistência à saúde nos programas do Ministério da Saúde (MS), educação em serviço e capacitação das equipes de saúde, planejamento, supervisão e avaliação dos serviços, assessoria técnica, atenção às urgências e emergências, educação para a comunidade e educação em saúde, assistência especializada ambulatorial, atividades administrativas, contratação e capacitação de recursos humanos e sistema de informação.

Dentre o conjunto de atividades do enfermeiro gerente, $28(63,6 \%)$ são de assistência à saúde nos programas do MS e $20(45,4 \%)$ de planejamento, supervisão, avaliação dos serviços e assessoria técnica nas unidades de saúde, o que representa as atividades de gerenciamento dos serviços. Em $16(36,3 \%)$ unidades de saúde são desenvolvidas outras atividades administrativas, contratação e capacitação de recursos humanos.

Em $13(29,5 \%)$ serviços são desenvolvidas ações de educação para a comunidade e 12 $(27,3 \%)$ ações de educação em serviço e capacitação para a equipe de saúde. Em 10 (22,7\%) unidades de saúde presta-se atendimento às urgências e emergências e 10 (22,7\%) assistência especializada ambulatorial.

Outros estudos também sinalizam que o enfermeiro desenvolve atividades simultâneas de assistência e de gerência. As atividades assistenciais de maior freqüência consistem no desenvolvimento de políticas de ações programáticas, tais como: saúde do adolescente, mulher, criança, adulto, idoso, saúde mental, tuberculose, entre outros programas do MS. Porém as atividades de gerência constituem em sua maioria, atividades administrativas burocráticas mais ligadas as questões operacionais do trabalho nas unidades de saúde. ${ }^{17}$ Em outro contexto, as ações administrativas são compostas de um conjunto de atividades voltadas para a organização do processo de trabalho, infraestrutura e planejamento de serviços. ${ }^{18}$

\section{$O$ trabalho gerencial dos enfermeiros}

Dentre o conjunto de atividades que os enfermeiros gerentes desenvolvem em um dia de trabalho, $61,4 \%$ delas são essencialmente de caráter gerencial, mas em $38,6 \%$ dos serviços eles desenvolvem simultaneamente atividades gerenciais e assistenciais, muitas vezes gerando conflitos diante da necessidade de articular ambos os tipos de ações.

Em estudo realizado no Rio Grande do Norte foi encontrada situação semelhante, com a maioria das enfermeiras, gerentes das UBSs, queixando-se de uma sobrecarga de ações administrativas. ${ }^{18}$ Elas ficam diante do difícil ou impossível impasse de buscar uma articulação entre a dimensão assistencial e a dimensão gerencial.

$\mathrm{Na}$ perspectiva dos enfermeiros que participaram dessa pesquisa, as atividades gerenciais consideradas de maior importância estão representadas na Figura 1, dispostas em ordem decrescente. A atividade gerencial de maior importância relacionada pelos enfermeiros foi promover a integração e o bom relacionamento com as equipes de saúde sob sua responsabilidade. Muitos gerentes apontaram como sendo fundamental investir no relacionamento interpessoal com sua equipe e valorizar o desempenho da equipe de saúde para o bom andamento e qualidade do cuidado prestado.

A competência interpessoal inclui habilidades como a flexibilidade, o espírito inovador e a criatividade, dar e receber informação, exercitar o relacionamento entendido na dimensão emocional afetiva. Esse processo precisa ser treinado juntamente com o crescimento pessoal que requer autopercepção, autoconscientização e autoaceitação. Pode ser uma ferramenta poderosa para transformar a prática gerencial do enfermeiro, uma vez que estimula a formação de líderes que possam avaliar e dimensionar os problemas de modo global, permitindo exercer o papel com mais segurança e transparência. ${ }^{14,19-20}$ 


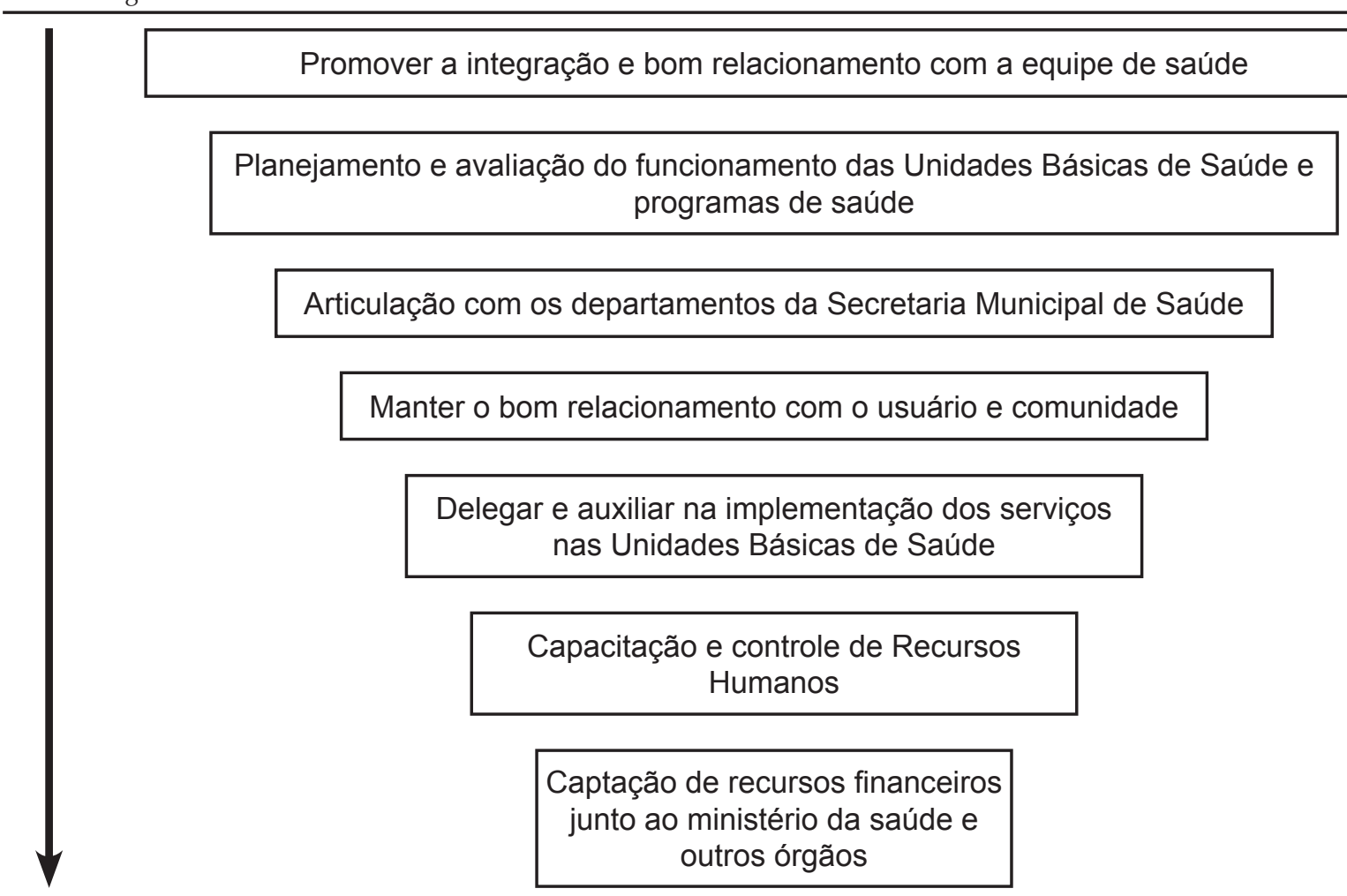

Figura 1 - Atividades gerenciais desenvolvidas pelos enfermeiros na Rede Básica de Saúde. Goiânia - GO, 2008

Outras atividades consideradas importantes no processo gerencial são o planejamento e a avaliação do funcionamento das UBSs, juntamente com os Programas de Saúde do Ministério implantados nestes serviços, além da articulação com os diversos departamentos da SMS. Neste caso, devemos considerar o princípio doutrinário do SUS de hierarquização, entendido como um conjunto articulado e contínuo das ações e serviços individuais e coletivos, em todos os níveis de complexidade do sistema. ${ }^{12}$

Os enfermeiros participantes da pesquisa consideram que, dentro do processo de trabalho gerencial, manter o bom relacionamento com o usuário e com a comunidade faz parte da função gerencial como uma ação de respeito à cidadania $\mathrm{e}$ participação popular. Isso levou-nos a considerar que de alguma forma os princípios do SUS estão norteando as ações dos gerentes no que diz respeito à participação da comunidade e à divulgação de informações quanto à capacidade e oferta dos serviços de saúde que o usuário tem como direito previsto nas Leis Orgânicas de Saúde. ${ }^{12,21}$

No entanto, o cuidado com a comunidade é uma preocupação a mais na função gerencial, somada às funções de organizar internamente a unidade de saúde e atender às necessidades de saúde da comunidade/usuários das áreas de abrangência. A proximidade com a comunidade estabelece relações e vínculos entre a comunidade e os serviços de saúde, mas, ao mesmo tempo, expõe as contradições presentes no cotidiano de trabalho, uma vez que outras necessidades surgirão para além do atendimento médico assistencial. ${ }^{22}$

Ainda no que se refere ao grupo de atividades consideradas importantes na função gerencial, os enfermeiros destacaram a ação de delegar e auxiliar na implementação dos serviços das UBSs, bem como a capacitação, controle de recursos humanos e a captação de recursos financeiros junto ao MS e outros órgãos (Figura 1).

Quanto à capacitação e controle de recursos humanos, a Portaria GM 1996, de 20 de agosto de 2007, instituiu a Política Nacional de Educação Permanente em Saúde como estratégia do SUS para a formação e o desenvolvimento de trabalhadores estruturados a partir da problematização da sua realidade de trabalho e que tenham como objetivo a transformação e organização das práticas profissionais, tendo como referência as necessidades dos usuários, das comunidades e do controle social em saúde. ${ }^{23}$ 
Também identificamos neste estudo, os pontos de estrangulamento presentes no cotidiano do trabalho gerencial do enfermeiro, que foram considerados fatores dificultadores no processo de trabalho gerencial. Sob muitos aspectos interferem para a baixa resolubilidade e queda da qualidade da assistência, gerando um sentimento de "frustração e/ou incapacidade" no desempenho da função gerencial.

A Figura 2, mostra em ordem decrescente, o conjunto de situações consideradas pontos de estrangulamentos no processo de trabalho gerencial.

A deficiência de recursos materiais, insumos e equipamentos foi considerada pelos enfer- meiros como o ponto de estrangulamento mais presente no trabalho gerencial. Isso em muitos momentos torna o atendimento inadequado, gerando a insatisfação do usuário, sobrecarga e estresse da equipe de saúde com queda de rendimento, tornando a função gerencial ainda mais conflituosa e desgastante para o profissional em seu cotidiano. Esses profissionais exercem uma função tensa e estafante, "que tem menos a ver com construir uma estrada em terreno firme e mais com buscar uma trilha à beira de areia movediça (...)" ${ }^{6: 21}$ Vivem pressionados para adotar ações de curto prazo na busca de recursos e solução de problemas.

Deficiência de recursos materiais, insumos e equipamentos

Trabalho desintegrado na rede de saúde

Falta de um plano de cargos carreiras e salários ou execução dele

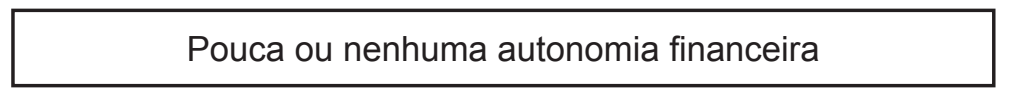

Grande número de reuniões

Demanda reprimida de especialidades

Figura 2 - Pontos de estrangulamento identificados pelos enfermeiros gerentes da Rede Básica de Saúde. Goiânia - GO, 2008

Para alguns enfermeiros gerentes, a insuficiência de recursos nem sempre causa impactos negativos. Em algumas situações pode ajudar os trabalhadores na tomada de decisão para resolução de problemas, auxiliando a equipe de saúde a descobrir maneiras de superar limites e promover parcerias de apoio entre os seus membros.

Isso mostra que as organizações de saúde não produzem serviços eficazes apenas com estruturas e pessoas. Os suprimentos em geral também fazem parte desse processo. A gestão eficaz de suprimentos irá fornecer materiais, insumos e equipamentos necessários, exercendo papel central na busca de um serviço eficiente de saúde para a população. ${ }^{24}$

No intuito de solucionar as deficiências de recursos os enfermeiros gerentes, tanto nas UBSs, nos CSs, nos DSs ou na SS em nível central, tomam providências semelhantes para suprir as necessidades de materiais, insumos e equipamentos, como o remanejamento interno ou com outros serviços de 
saúde, improvisação e/ou por meio de solicitação aos departamentos competentes nos processos de licitações ou de compras emergenciais.

Portanto, se por um lado podemos tratar a gerência como científica racional, com possibilidade de prever e antecipar ações mais eficientes e conseqüentes, por outro, há na gerência, "[...] uma face de imprevisibilidade e de interação humana que lhe confere a dimensão do ilógico, do intuitivo, do emocional e espontâneo e do irracional" .6:25

O trabalho com pouca integração na RBS foi considerado pelos enfermeiros como o segundo ponto de estrangulamento mais presente no trabalho gerencial, principalmente por parte dos gerentes lotados em UBSs, Unidades de Saúde da Família (USFs), CSs e em alguns DSs. Consideram moroso o respaldo do nível central em atender às demandas em algumas situações que exigem posicionamentos e respostas mais rápidas.

Por outro lado, os enfermeiros que gerenciam ou coordenam em nível central sentem-se impossibilitados de apoiar os serviços com maior agilidade, pois, em algumas circunstâncias, o poder de tomada de decisão é limitado e muitas vezes dependem de outros departamentos.

Entre os fatores evidenciados pelos enfermeiros para a desintegração no trabalho gerencial esta a dificuldade de comunicação entre os vários níveis do sistema de saúde, fato este caracterizado, principalmente, pela duplicidade de informações, com conteúdos diferentes ou pela falta de informação, gerando insegurança e conflitos. Outro estudo também aponta as dificuldades de comunicação entre os DSs, unidades de saúde da família, coordenadores e população, como um desafio que merece ser superado. ${ }^{17}$

Outro aspecto que leva à pouca integração é a baixa resolutividade e autonomia, uma vez que, na proposta de consolidação do SUS, há toda uma regulamentação no sentido da operacionalizar os serviços prestados, mas não há, ao mesmo tempo, a efetivação do processo de descentralização ao nível mais operacional, parecendo haver uma incoerência entre os propósitos de descentralizar as ações de saúde e a forma de organizar os serviços na RBS.

A gestão hierarquizada e verticalizada cria dificuldades gerenciai e fragmentação do processo de trabalho em quase todas as áreas. Para que ocorra de fato um trabalho integrado que contribua para aumentar o impacto das ações é importante não somente facilitar a comunicação entre as várias unidades de saúde, setores e níveis gerenciais, mas adotar um sistema que produza um compartilha- mento simultâneo de responsabilidades pelas ações sistemáticas com definição de papéis. ${ }^{25-26}$

Um outro ponto de estrangulamento considerado importante para alguns enfermeiros é a falta de um Plano de Carreiras, Cargos e Salários (PCCS) ou, segundo outros, a sua execução. Constatamos essa dificuldade quando questionamos os enfermeiros gerentes a respeito da existência de um PCCS, $59,1 \%$ responderam não existir ou desconhecerem qualquer política neste sentido, enquanto 40,9\% dos enfermeiros afirmaram que existe uma política de cargos e salários, porém muito incipiente. Estes informaram ainda que o conteúdo dessa proposta não é muito divulgado ou têm pouca informação sobre um PCCS a que estão submetidos.

OPCCS foi apontado pelos dirigentes de recursos humanos das Secretarias Estaduais como o mais sério problema para a gestão de recursos humanos nos Estados, além de contribuir para o surgimento de insatisfação entre os servidores públicos. ${ }^{27}$

Há um consenso entre os secretários estaduais da necessidade de valorizar a carreira e não se limitar a ser apenas um plano de cargos e/ou um plano de salários. As Diretrizes Nacionais para a instituição de PCCS no âmbito do SUS foi aprovado em novembro de 2006 e entregue ao Conselho Nacional de Saúde que orienta a criação ou reforma de planos de carreiras, cargos e salários. ${ }^{12,27}$

No que diz respeito à autonomia financeira como um ponto de estrangulamento, a maioria dos enfermeiros respondeu não ter nenhuma governabilidade nesse nível de participação. Percebemos que a falta de autonomia dos gerentes de serviços públicos de saúde vai muito além das questões financeiras, pois os profissionais de saúde com funções gerenciais possuem uma autonomia relativa para o desenvolvimento de suas ações. ${ }^{28}$

Os enfermeiros gerentes lotados em nível central também evidenciaram que o grande número de reuniões locais com o nível central pode ser considerado um ponto de estrangulamento, pois estão constantemente reunindo-se com diversas equipes de serviço da RBS e isso dificulta a continuidade das atividades programadas, tornando o trabalho moroso e às vezes interferindo na qualidade do cuidado com a equipe e usuários.

Oúltimo ponto de estrangulamento apontado pelos enfermeiros foi o atendimento aos usuários com uma demanda reprimida de especialidades, o que muitas vezes interrompe o tratamento e fere os princípios do SUS da integralidade e da eqüidade. A integralidade não é apenas uma diretriz definida constitucionalmente, significa considerar a pessoa 
como um todo, devendo as ações de saúde procurar atender a todas as suas necessidades. ${ }^{29}$ A eqüidade é entendida como um princípio de justiça social que garante a igualdade da assistência à saúde, sem preconceitos ou privilégios de qualquer espécie. A rede de serviços deve estar atenta às necessidades reais da população a ser atendida. ${ }^{12}$

Há evidências de que o volume de serviços oferecidos à população brasileira ainda é insuficiente. Por isso é necessário reconhecer que a demanda reprimida de especialidades também é insuficiente para atender com qualidade o usuário, o que se torna um fator dificultador para o gerenciamento dos serviços. Mas, de qualquer modo, esses recursos poderiam ter um uso mais adequado e racional, caso ocorressem reordenações no modelo de gestão e de atenção. ${ }^{25}$

\section{CONCLUSÃO}

A contribuição dos enfermeiros dos serviços públicos de saúde, com experiência em gerenciamento, foi de grande significado para repensarmos a formação desse profissional em nível de graduação e subsidiarmos os docentes da área de Saúde Pública dos Cursos de Enfermagem para um enfoque que privilegie a gestão pública nos serviços de saúde.

Assim, acreditamos que as competências e habilidades específicas para a área de administração e processo gerencial em enfermagem são desenvolvidas durante o processo de formação, sendo permanentemente construídas nas experiências cotidianas. As experiências podem colaborar na capacitação do profissional para avaliar, sistematizar e decidir as condutas mais adequadas, com base em evidências científicas. Podem ainda desenvolver o espírito de liderança no trabalho em equipe, comunicação para interagir com a equipe e público em geral e gerenciamento da força de trabalho dos recursos físicos, materiais e informação, entre outras ferramentas capazes de instrumentalizar o trabalho gerência. ${ }^{30}$

Neste sentido, uma das importantes interseções entre saúde e educação, diz respeito à adequação do ensino, conhecimentos produzidos e serviços prestados à população com base nas necessidades sociais, mas tendo como eixo norteador as Diretrizes Curriculares Nacionais do Curso de Graduação em Enfermagem de 2001, que preconiza como perfil do profissional Enfermeiro uma formação generalista, humanista, crítica e reflexiva.
Para a construção desse perfil profissional, há necessidade de o ensino fortalecer parcerias entre ensino e serviço, no sentido de dar sustentação à formação gerencial do enfermeiro numa perspectiva transformadora, e buscar novos cenários para a formação, como as USFs. Para isso é imprescindível o papel do docente como facilitador no processo ensino-aprendizagem e articulador na integração da teoria com a pratica, em que o enfermeiro do serviço compartilha com ambos suas necessidades e desafios.

\section{REFERENNCIAS}

1. Witt RR. Competências da enfermeira na atenção básica: contribuição das Funções Essenciais de Saúde Pública [tese]. Ribeirão Preto (SP): Universidade de São Paulo. Programa de Pós-Graduação em Enfermagem da Escola de Enfermagem de Ribeirão Preto; 2005.

2. Bertussi DC, Almeida MJ. A influência de um projeto de intervenção no campo de recursos humanos em saúde: Projeto Gerus. Brasília (DF): Ministério da Saúde; 2003.

3. Felli VEA, Peduzzi M. O trabalho gerencial em enfermagem. In: Kurcgant, P. organizador. Gerenciamento em Enfermagem. Rio de Janeiro (RJ): Guanabara Koogan; 2005. p.1-13.

4. Trevizan MA, Mendes IAC, Melo MRA. Liderança e comunicação no cenário da gestão em enfermagem. Rev Latino-am Enfermagem. 1998 Dez; 6(5):77-82.

5. Machado MH. Gestão do trabalho em saúde no contexto de mudanças. RAP. 2000 Jul-Ago; 34(4):133-46.

6. Motta PR. Gestão Contemporânea: A ciência e a arte de ser dirigente. $14^{\mathrm{a}}$ ed. Rio de Janeiro (RJ): Record; 2003.

7. Campos GWS. Subjetividade e administração de pessoal: considerações sobre modelos de gerenciar o trabalho em equipe de saúde. In: Merhy EE, Onocko $\mathrm{R}$, organizadores. Agir em Saúde um desafio para o público. $2^{\mathrm{a}}$ ed. São Paulo (SP): Hucitec; 2002.

8. Merhy EE, Junior HMM, Rimolli J, Franco TB, Bueno WS. O trabalho em saúde: olhando e experienciando o SUS no cotidiano. $3^{\text {a }}$ ed. São Paulo (SP): Hucitec; 2006.

9. Munari DB, Cruz RMM, Merjane TVB. A aplicação do modelo de educação de laboratório no processo de formação do enfermeiro. Rev Enferm UERJ. 2005 Maio-Ago; 13(2):263-9.

10. Munari DB, Rocha BS, Medeiros M, Bezerra ALQ, Barbosa MA, Weirich CF. O modelo de educação de laboratório na formação do enfermeiro: estudo com egressos. Cienc Cuid Saude. 2008 Jan-Mar; 7(1):89-97. 
11. Conselho Nacional de Educação (BR). Parecer do CNE/CES no1133 de 7 de agosto de 2001. Dispõe sobre as Diretrizes Curriculares Nacionais dos Cursos de Graduação em Enfermagem, Medicina e Nutrição. Brasília (DF); 2001.

12. Ministério da Saúde (BR), Conselho Nacional de Secretários de Saúde. Sistema Único de Saúde. Brasília (DF): MS/CONASS; 2007.

13. Bezerra ALQ. Ocontexto da educação continuada em enfermagem na visão dos gerentes de enfermagem e enfermeiros de educação continuada [tese]. São Paulo (SP): Universidade de São Paulo. Programa de Pós-Graduação em Enfermagem; 2000.

14. Munari, DB; Bezerra, ALQ. Inclusão da competência interpessoal na formação do enfermeiro como gestor. Rev Bras Enferm. 2004 Jul-Ago; 57(4):484-6.

15. Gil, AC. Como elaborar projetos de pesquisa. $4^{\mathrm{a}} \mathrm{ed}$. São Paulo (SP): Atlas; 2002.

16. Secretaria Municipal de Saúde de Goiânia. Departamento de Rede Básica, Documento técnico: Diretrizes da Secretaria Municipal de Goiânia para o trabalho das equipes de Saúde da Família. Goiânia (GO): SMS; 2006.

17. Kantorski LP, Jardim VMR, Coimbra VCC, Oliveira MM, Heck RM. Integralidade da atenção à saúde na perspectiva da gestão no município. Texto Contexto Enferm. 2006 Jul-Set; 15(3):434-41.

18. Vanderlei, MIG. O gerenciamento na estratégia da saúde da família: o processo de trabalho dos gestores e dos gerentes municipais de saúde no estado do Maranhão [tese]. Ribeirão Preto (SP): Universidade deSão Paulo. Programa de Pós-Graduação em Enfermagem da Escola de Enfermagem de Ribeirão Preto; 2005.

19. Munari DB, Costa HK, Cardoso AHA, Almeida CCOF. Características da competência interpessoal do enfermeiro: estudo com graduandos de enfermagem. Rev Bras Enferm. 2003 Set-Out; 56(5):484-7.

20. Jorge MSB, Freitas CHA, Nobrega MFB, Queiroz MVO. Gerenciamento em enfermagem: um olhar crítico sobre o conhecimento produzido em periódicos brasileiros (2000-2004). Rev Bras Enferm. 2007 Jan-Fev; 60(1):81-6.
21. Carvalho GI, Santos L. Sistema Único de Saúde Comentários à Lei Orgânica da Saúde (Leis 8.080/90 e 8.142/90). $2^{a}$ ed. São Paulo (SP): Hucitec; 1995.

22. Mishima SM. Constituição do gerenciamento local na RBS em Ribeirão Preto [tese]. Ribeirão Preto (SP): Universidade de São Paulo. Programa de Pós-Graduação em Enfermagem da Escola de Enfermagem de Ribeirão Preto; 1995.

23. Almeida LPG, Ferraz CA. Políticas de formação de recursos humanos em saúde e enfermagem. Rev Bras Enferm. 2008 Jan-Fev: 61(1):31-5.

24. Cruz ACFV, Henriques MGP. A gestão de suprimentos nos centros públicos de saúde da Prefeitura Municipal de Salvador [online]. RAU. 2005 [acesso em 2009 Abr 17]; 4(4):11-52. Disponível em: http:/ / www.unime.com.br/2006/rau/4/arquivos/RAU_ v4n4_jundez2005_02_CRUZ_HENRIQUES_A\%20 Gest\%E3o\%20de\%20suprimentos\%20nos\%20centros $\% 20 \mathrm{p} \%$ FAblicos $\% 20 \mathrm{de} \% 20$ sa $\%$ FAde $\% 20 \mathrm{da} \% 20$ Prefeitura\%20Municipal\%20de\%20Salvador.pdf.

25. Campos GWS, Domotti, AC. Apoio matricial e equipes de referência: uma metodologia para gestão do trabalho interdisciplinar em saúde. Cad Saúde Pública. 2007 Fev; 23(2):399-407.

26. Campos GWS, Minayo MCS, Akerman M, Júnior MD, Carvalho, YM. organizadores. Tratado de Saúde Coletiva, $2^{a}$ ed. São Paulo/Rio de Janeiro (SP/RJ): Hucitec/Fiocruz; 2007.

27. Ministério da Saúde (BR), Conselho Nacional de Secretários de Saúde. Gestão do Trabalho na Saúde. Brasília (DF): MS/CONASS; 2007.

28. Abrahão AL, Martins $\mathrm{CM}$, Geisler A. O sujeito na literatura acadêmica sobre gestão em saúde: notas para a questão da autonomia. Ciênc Saúde Colet. 2008 Jan-Fev; 13(1):247-57.

29. Pinheiro R, Mattos RA. organizadores. Os sentidos da integralidade na atenção e no cuidado à saúde. $6^{\mathrm{a}}$ ed. Rio de Janeiro (RJ): CEPES-ABRASCO; 2006.

30. Vale EG, Guedes MVC. Competências e habilidades no ensino de administração em enfermagem à luz das diretrizes curriculares nacionais. Rev Bras Enferm. 2004 Jul-Ago; 57(4):475-8. 